\title{
Crystal structure of $6,6^{\prime}$-ditosyl- $\alpha-\alpha^{\prime}$-trehalose-methanol-water $(1 / 2 / 2)$, $\mathrm{C}_{52} \mathrm{H}_{68} \mathrm{O}_{30} \mathrm{~S}_{4} \cdot 2 \mathrm{CH}_{3} \mathrm{OH} \cdot 2 \mathrm{H}_{2} \mathrm{O}$
}

\author{
M. Saviano*, , R. Iacovino ${ }^{\mathrm{I}}$, E. Benedetti ${ }^{\mathrm{I}}$, V. Cucinotta ${ }^{\mathrm{ll}}$, G. Grasso ${ }^{\mathrm{Ill}}$ and D. Sciotto ${ }^{\mathrm{Il}}$ \\ I University of Naples, Department of Chemistry, Biocrystallography Research Centre of the C.N.R., via Mezzocannone 4, I-80I34 Napoli, Italy \\ II University of Catania, Dipartimento di Scienze Chimiche, viale A. Doria, 8, I-95I25 Catania, Italy \\ III Istituto per lo studio delle sostanze naturali di Interesse, Alimentare e Chimico Farmaceutico C.N.R., viale A. Doria, 8, I-95125 Catania, Italy
}

Received November 17, 1998, CCDC-No. $1267 / 63$

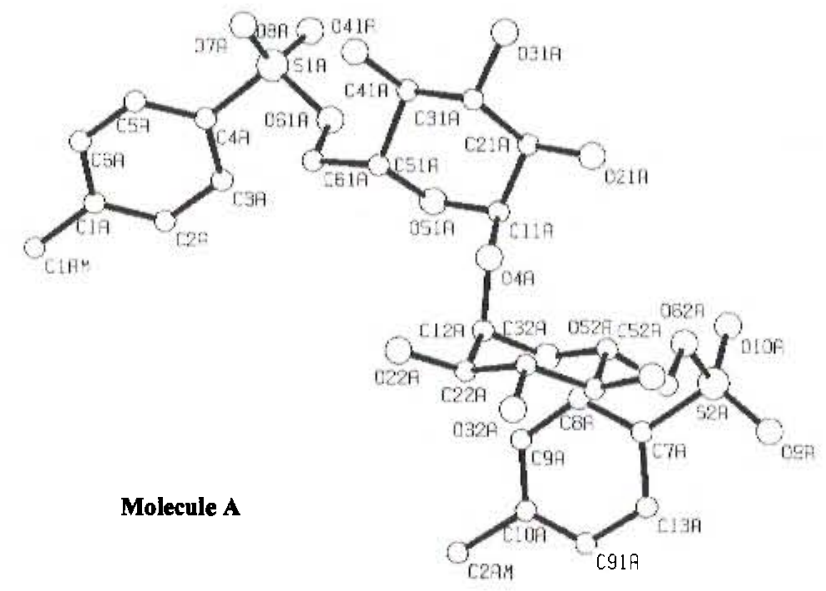

\begin{abstract}
Discussion
Hydrogen atom $s$ of the solvent molecules are not refined.

The $\alpha, \alpha^{\prime}$-trehalose is a peculiar disaccharide, in which two Dglucopyrasinic rings are linked by a bond between their anomeric carbon atoms, with a consequent non-reductive behaviour, while the molecule is symmetric. Owing to this peculiarity, this compound has attracted the interest of many reserchers and many derivatives have been synthesized. One of the key intermediates in these syntheses is the 6,6'-ditosyl-trehalose, which was first synthesized and characterized in 1958 [2]. The two independent molecules are almost superimposable with angle between the planes of the two glucosidic rings of $46.5(4)^{\circ}$ and $53.5(5)^{\circ}$ for molecule A and B, respectively. The major difference between the two independent molecules is represented by one dihedral angles in one tosyl moiety $\left(\mathrm{C} 62 \mathrm{~A}-\mathrm{O} 62 \mathrm{~A}-\mathrm{S} 2 \mathrm{~A}-\mathrm{C} 7 \mathrm{~A}=69(1)^{\circ}\right.$ and $\left.\mathrm{C} 63 \mathrm{~B}-\mathrm{O} 63 \mathrm{~B}-\mathrm{S} 1 \mathrm{~B}-\mathrm{C} 4 \mathrm{~B}=177.1(8)^{\circ}\right)$.

Table 1. Data collection and handling.

$\begin{array}{ll}\text { Crystal: } & \text { colorless needle, size } 0.2 \times 0.3 \times 0.6 \mathrm{~mm} \\ \text { Wavelength: } & \text { Cu } K_{\alpha} \text { radiation }(1.54056 \AA) \\ \mu: & 21.30 \mathrm{~cm}^{-1} \\ \text { Diffractometer, scan mode: } & \text { Nonius CAD4-Turbo, } 0 / 2 \theta \\ 2 \hat{\theta}_{\text {max }}: & 140.22^{\circ} \\ N(h k l)_{\text {measured }}, N(h k l)_{\text {unique: }}: & 6654,6654 \\ \text { Criterion for } l_{\text {obs }}, N(h k l)_{\text {gl }}: & I_{\text {obs }}>2 \sigma\left(I_{\text {obs }}\right), 3015 \\ N(\text { param) } & 830 \\ \text { Programs: } & \text { SDP [3], SIR92 [4], SHELXL-93 [5], } \\ & \text { PLATON [6] }\end{array}$
\end{abstract}

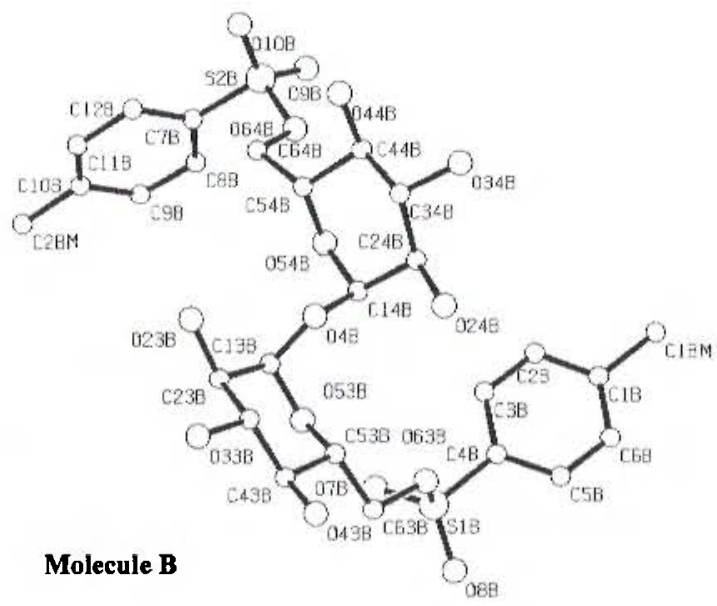

Täble 2. Atomic coordinates and displacement parameters (in $\AA^{2}$ ).

\begin{abstract}
$\mathrm{C}_{54} \mathrm{H}_{80} \mathrm{O}_{32} \mathrm{~S}_{4}$, monoclinic, $P 12_{1} \mathrm{l}$ (No. 4), $a=17.334(6) \AA$, $b=7.814(6) \AA, c=24.278(6) \AA, \beta=99.82(2)^{\circ}, V=3240.2 \AA^{3}$, $Z=2, R_{\mathrm{gt}}(F)=0.083, R_{\mathrm{w}}\left(F^{2}\right)=0.203, T=293 \mathrm{~K}$.
\end{abstract}

\section{Source of material}

The title compound was obtained by a one-step synthesis [1]. In order to select the primary site, the most reactive, the reaction was carried out at low temperature, followed by an accurate purification by reverse-phase chromatography, thus obtaining the product in short time and in good yield. The compound was crystallised from methanol and water mixture and is characterized by two independent molecules (A and B) in the asymmetric unit.

* Correspondence author (e-mail: SAVIANO@CHEMNA.DICHI.UNINA.IT)

\begin{tabular}{llllll}
\hline Atom & Site & $x$ & $y$ & $z$ & $U_{\text {iso }}$ \\
\hline $\mathrm{H}(1 \mathrm{M} 1)$ & $2 a$ & $0.961(1)$ & $-0.877(2)$ & $0.8661(8)$ & 0.229 \\
$\mathrm{H}(1 \mathrm{M} 2)$ & $2 a$ & $0.957(1)$ & $-0.991(2)$ & $0.8126(8)$ & 0.229 \\
$\mathrm{H}(1 \mathrm{M} 3)$ & $2 a$ & $0.888(1)$ & $-0.997(2)$ & $0.8467(8)$ & 0.229 \\
$\mathrm{H}(2 \mathrm{~A})$ & $2 a$ & $0.940(1)$ & $-0.591(2)$ & $0.8453(6)$ & 0.124 \\
$\mathrm{H}(3 \mathrm{~A})$ & $2 a$ & $0.8802(8)$ & $-0.366(2)$ & $0.7917(6)$ & 0.104 \\
$\mathrm{H}(5 \mathrm{~A})$ & $2 a$ & $0.7633(9)$ & $-0.713(2)$ & $0.6882(6)$ & 0.103 \\
$\mathrm{H}(6 \mathrm{~A})$ & $2 a$ & $0.8251(9)$ & $-0.929(2)$ & $0.7395(8)$ & 0.127 \\
$\mathrm{H}(61 \mathrm{~A})$ & $2 a$ & $0.7085(7)$ & $-0.296(1)$ & $0.8037(5)$ & 0.081 \\
$\mathrm{H}(61 \mathrm{~B})$ & $2 a$ & $0.6525(7)$ & $-0.352(1)$ & $0.7487(5)$ & 0.081 \\
$\mathrm{H}(51 \mathrm{~A})$ & $2 a$ & $0.5939(7)$ & $-0.128(1)$ & $0.7919(4)$ & 0.076 \\
$\mathrm{H}(41 \mathrm{~A})$ & $2 a$ & $0.6380(7)$ & $-0.002(1)$ & $0.6907(4)$ & 0.068 \\
$\mathrm{H}(41 \mathrm{~B})$ & $2 a$ & $0.5749(4)$ & $-0.2439(9)$ & $0.6781(3)$ & 0.101 \\
$\mathrm{H}(31 \mathrm{~A})$ & $2 a$ & $0.5086(7)$ & $0.093(1)$ & $0.7391(4)$ & 0.078 \\
$\mathrm{H}(31 \mathrm{~B})$ & $2 a$ & $0.5264(5)$ & $0.143(1)$ & $0.6404(3)$ & 0.117 \\
$\mathrm{H}(21 \mathrm{~A})$ & $2 a$ & $0.6414(7)$ & $0.290(1)$ & $0.7313(4)$ & 0.069 \\
$\mathrm{H}(21 \mathrm{~B})$ & $2 a$ & $0.5379(5)$ & $0.4448(9)$ & $0.7327(3)$ & 0.114 \\
\hline
\end{tabular}


Table 2. Continued.

\begin{tabular}{llllll}
\hline Atom & Site & $x$ & $y$ & $z$ & $U_{\text {iso }}$ \\
\hline H(11A) & $2 a$ & $0.6859(7)$ & $0.270(1)$ & $0.8227(4)$ & 0.073 \\
H(12A) & $2 a$ & $0.6631(9)$ & $0.055(2)$ & $0.9049(5)$ & 0.101 \\
H(22A) & $2 a$ & $0.586(1)$ & $0.097(2)$ & $0.9710(6)$ & 0.118 \\
H(22B) & $2 a$ & $0.4927(7)$ & $-0.073(1)$ & $0.9339(5)$ & 0.175 \\
H(32A) & $2 a$ & $0.475(1)$ & $0.263(2)$ & $0.8879(6)$ & 0.11 \\
H(32B) & $2 a$ & $0.4215(6)$ & $0.133(1)$ & $0.9511(4)$ & 0.164 \\
H(42A) & $2 a$ & $0.566(1)$ & $0.407(2)$ & $0.9854(6)$ & 0.113 \\
H(42B) & $2 a$ & $0.5078(6)$ & $0.625(1)$ & $0.9153(5)$ & 0.174 \\
H(52A) & $2 a$ & $0.5931(9)$ & $0.472(2)$ & $0.8751(5)$ & 0.094 \\
H(62A) & $2 a$ & $0.6266(8)$ & $0.689(2)$ & $0.9445(5)$ & 0.096 \\
H(62B) & $2 a$ & $0.6830(8)$ & $0.558(2)$ & $0.9802(5)$ & 0.096 \\
H(8A) & $2 a$ & $0.834(1)$ & $0.367(2)$ & $0.9148(7)$ & 0.124 \\
H(9A) & $2 a$ & $0.891(1)$ & $0.137(3)$ & $0.9673(9)$ & 0.144 \\
H(91A) & $2 a$ & $0.909(1)$ & $0.400(3)$ & $1.1052(7)$ & 0.139 \\
H(13A) & $2 a$ & $0.855(1)$ & $0.643(2)$ & $1.0569(7)$ & 0.127 \\
H(2M1) & $2 a$ & $0.945(1)$ & $-0.003(3)$ & $1.048(1)$ & 0.263 \\
H(2M2) & $2 a$ & $0.905(1)$ & $0.053(3)$ & $1.098(1)$ & 0.263 \\
H(2M3) & $2 a$ & $0.991(1)$ & $0.112(3)$ & $1.095(1)$ & 0.263 \\
H(1M4) & $2 a$ & $0.070(1)$ & $1.196(3)$ & $0.6196(8)$ & 0.212 \\
H(1M5) & $2 a$ & $0.031(1)$ & $1.297(3)$ & $0.5663(8)$ & 0.212 \\
H(1M6) & $2 a$ & $0.121(1)$ & $1.320(3)$ & $0.5896(8)$ & 0.212 \\
H(2B) & $2 a$ & $0.088(1)$ & $0.901(2)$ & $0.6023(7)$ & 0.136 \\
H(3B) & $2 a$ & $0.118(1)$ & $0.680(2)$ & $0.5467(7)$ & 0.141 \\
H(5B) & $2 a$ & $0.1408(9)$ & $1.016(2)$ & $0.4267(6)$ & 0.107 \\
H(6B) & $2 a$ & $0.1070(7)$ & $1.232(2)$ & $0.4810(7)$ & 0.106 \\
H(63A) & $2 a$ & $0.3106(7)$ & $0.550(2)$ & $0.4092(5)$ & 0.081 \\
& & & & &
\end{tabular}

Table 2. Continued.

\begin{tabular}{llclll}
\hline Atom & Site & $x$ & $y$ & $z$ & $U_{\text {iso }}$ \\
\hline H(63B) & $2 a$ & $0.2399(7)$ & $0.425(2)$ & $0.4117(5)$ & 0.081 \\
H(53B) & $2 a$ & $0.3580(6)$ & $0.484(1)$ & $0.5067(4)$ & 0.067 \\
H(43B) & $2 a$ & $0.3478(7)$ & $0.190(1)$ & $0.4368(4)$ & 0.069 \\
H(43A) & $2 a$ & $0.4770(5)$ & $0.333(1)$ & $0.4449(3)$ & 0.101 \\
H(33B) & $2 a$ & $0.4572(7)$ & $0.260(1)$ & $0.5341(4)$ & 0.074 \\
H(33A) & $2 a$ & $0.4658(5)$ & $0.038(1)$ & $0.4618(3)$ & 0.118 \\
H(23B) & $2 a$ & $0.3356(7)$ & $0.009(1)$ & $0.5225(4)$ & 0.073 \\
H(23A) & $2 a$ & $0.4360(5)$ & $-0.0467(9)$ & $0.5876(3)$ & 0.106 \\
H(13B) & $2 a$ & $0.2671(6)$ & $0.175(1)$ & $0.5750(4)$ & 0.066 \\
H(14B) & $2 a$ & $0.2547(7)$ & $0.500(1)$ & $0.5877(5)$ & 0.08 \\
H(24B) & $2 a$ & $0.3023(7)$ & $0.700(1)$ & $0.6523(5)$ & 0.078 \\
H(24A) & $2 a$ & $0.4108(5)$ & $0.7682(9)$ & $0.6213(3)$ & 0.107 \\
H(34B) & $2 a$ & $0.4363(7)$ & $0.502(1)$ & $0.6936(5)$ & 0.076 \\
H(34A) & $2 a$ & $0.4448(5)$ & $0.782(1)$ & $0.7058(3)$ & 0.133 \\
H(44B) & $2 a$ & $0.3103(8)$ & $0.544(1)$ & $0.7507(5)$ & 0.083 \\
H(44A) & $2 a$ & $0.4472(6)$ & $0.446(1)$ & $0.7845(3)$ & 0.139 \\
H(54B) & $2 a$ & $0.3536(7)$ & $0.252(1)$ & $0.6932(5)$ & 0.078 \\
H(64A) & $2 a$ & $0.2457(8)$ & $0.105(2)$ & $0.7140(5)$ & 0.095 \\
H(64B) & $2 a$ & $0.3010(8)$ & $0.151(2)$ & $0.7704(5)$ & 0.095 \\
H(8B) & $2 a$ & $0.019(1)$ & $0.143(2)$ & $0.7210(7)$ & 0.118 \\
H(9B) & $2 a$ & $-0.035(1)$ & $-0.099(2)$ & $0.6764(6)$ & 0.123 \\
H(11B) & $2 a$ & $0.1469(9)$ & $-0.373(2)$ & $0.7467(8)$ & 0.133 \\
H(12B) & $2 a$ & $0.1982(9)$ & $-0.135(2)$ & $0.7984(7)$ & 0.123 \\
H(2M4) & $2 a$ & $-0.037(1)$ & $-0.377(2)$ & $0.6541(8)$ & 0.208 \\
H(2M5) & $2 a$ & $0.003(1)$ & $-0.502(2)$ & $0.7010(8)$ & 0.208 \\
H(2M6) & $2 a$ & $0.045(1)$ & $-0.454(2)$ & $0.6511(8)$ & 0.208 \\
\hline & & & & & \\
\hline
\end{tabular}

Table 3. Atomic coordinates and displacement parameters (in $\AA^{2}$ ).

\begin{tabular}{|c|c|c|c|c|c|c|c|c|c|c|}
\hline Atom & Site & $x$ & $y$ & $z$ & $U_{11}$ & $U_{22}$ & $U_{33}$ & $U_{12}$ & $U_{13}$ & $U_{23}$ \\
\hline $\mathrm{C}(\mathrm{IMA})$ & $2 a$ & $0.927(1)$ & $-0.925(2)$ & $0.8343(8)$ & $0.18(2)$ & $0.10(2)$ & $0.20(2)$ & $0.08(1)$ & $0.08(2)$ & $0.08(1)$ \\
\hline $\mathrm{C}(1 \mathrm{~A})$ & $2 a$ & $0.8869(9)$ & $-0.783(2)$ & $0.7991(7)$ & $0.10(1)$ & $0.055(9)$ & $0.13(1)$ & $-0.003(8)$ & $0.017(9)$ & $0.033(9)$ \\
\hline $\mathrm{C}(2 \mathrm{~A})$ & $2 a$ & $0.904(1)$ & $-0.615(2)$ & $0.8134(6)$ & $0.14(1)$ & $0.08(1)$ & $0.078(9)$ & $0.04(1)$ & $-0.005(8)$ & $0.013(8)$ \\
\hline $\mathrm{C}(3 \mathrm{~A})$ & $2 a$ & $0.8678(8)$ & $-0.478(2)$ & $0.7816(6)$ & $0.11(1)$ & $0.046(7)$ & $0.097(9)$ & $-0.013(7)$ & $-0.010(8)$ & $0.005(7)$ \\
\hline $\mathrm{C}(4 \mathrm{~A})$ & $2 a$ & $0.8119(8)$ & $-0.517(2)$ & $0.7335(6)$ & $0.087(8)$ & $0.039(6)$ & $0.091(8)$ & $0.004(6)$ & $0.020(7)$ & $-0.001(6)$ \\
\hline $\mathrm{C}(5 \mathrm{~A})$ & $2 a$ & $0.7990(9)$ & $-0.687(2)$ & $0.7202(6)$ & $0.11(1)$ & $0.033(7)$ & $0.11(1)$ & $0.003(7)$ & $0.018(8)$ & $-0.015(7)$ \\
\hline$C(6 A)$ & $2 a$ & $0.8352(9)$ & $-0.816(2)$ & $0.7510(8)$ & $0.11(1)$ & $0.048(9)$ & $0.16(2)$ & $0.016(8)$ & $0.03(1)$ & $-0.01(1)$ \\
\hline$S(1 \mathrm{~A})$ & $2 a$ & $0.7664(2)$ & $-0.3572(4)$ & $0.6923(1)$ & $0.102(2)$ & $0.058(2)$ & $0.078(2)$ & $0.014(2)$ & $0.020(2)$ & $0.007(2)$ \\
\hline$O(7 A)$ & $2 a$ & $0.6958(6)$ & $-0.428(1)$ & $0.6604(4)$ & $0.112(7)$ & $0.092(7)$ & $0.081(5)$ & $0.011(6)$ & $-0.004(5)$ & $-0.013(5)$ \\
\hline $\mathrm{O}(8 \mathrm{~A})$ & $2 a$ & $0.8217(6)$ & $-0.267(1)$ & $0.6647(4)$ & $0.138(8)$ & $0.088(8)$ & $0.128(8)$ & $0.032(7)$ & $0.070(7)$ & $0.055(7)$ \\
\hline $\mathrm{O}(61 \mathrm{~A})$ & $2 a$ & $0.7440(5)$ & $-0.2182(9)$ & $0.7346(3)$ & $0.095(6)$ & $0.036(4)$ & $0.093(6)$ & $0.000(4)$ & $0.033(5)$ & $-0.007(4)$ \\
\hline $\mathrm{C}(61 \mathrm{~A})$ & $2 a$ & $0.6848(7)$ & $-0.260(1)$ & $0.7665(5)$ & $0.099(8)$ & $0.039(6)$ & $0.068(7)$ & $0.012(6)$ & $0.027(6)$ & $-0.001(5)$ \\
\hline $\mathrm{C}(51 \mathrm{~A})$ & $2 a$ & $0.6349(7)$ & $-0.102(1)$ & $0.7700(4)$ & $0.111(9)$ & $0.019(5)$ & $0.056(6)$ & $-0.015(5)$ & $0.002(6)$ & $-0.001(5)$ \\
\hline $\mathrm{O}(51 \mathrm{~A})$ & $2 a$ & $0.6854(5)$ & $0.0290(9)$ & $0.7983(3)$ & $0.088(5)$ & $0.034(4)$ & $0.077(5)$ & $0.001(4)$ & $0.019(4)$ & $0.001(4)$ \\
\hline $\mathrm{C}(41 \mathrm{~A})$ & $2 a$ & $0.5964(7)$ & $-0.032(1)$ & $0.7117(4)$ & $0.083(7)$ & $0.033(5)$ & $0.052(5)$ & $-0.014(5)$ & $0.005(5)$ & $0.006(4)$ \\
\hline $\mathrm{O}(41 \mathrm{~A})$ & $2 a$ & $0.5486(4)$ & $-0.1586(9)$ & $0.6815(3)$ & $0.093(5)$ & $0.034(4)$ & $0.072(4)$ & $-0.013(4)$ & $0.007(4)$ & $-0.010(4)$ \\
\hline$C(31 \mathrm{~A})$ & $2 a$ & $0.5507(7)$ & $0.125(1)$ & $0.7188(4)$ & $0.109(9)$ & $0.025(5)$ & $0.060(6)$ & $-0.006(6)$ & $0.011(6)$ & $0.001(5)$ \\
\hline$O(31 \mathrm{~A})$ & $2 a$ & $0.5153(5)$ & $0.200(1)$ & $0.6663(3)$ & $0.115(6)$ & $0.041(4)$ & $0.070(5)$ & $-0.015(5)$ & $-0.007(4)$ & $0.012(4)$ \\
\hline $\mathrm{C}(21 \mathrm{~A})$ & $2 a$ & $0.6020(7)$ & $0.256(1)$ & $0.7538(4)$ & $0.085(7)$ & $0.028(5)$ & $0.058(6)$ & $0.000(5)$ & $0.008(5)$ & $0.005(4)$ \\
\hline $\mathrm{O}(21 \mathrm{~A})$ & $2 a$ & $0.5618(5)$ & $0.4096(9)$ & $0.7627(3)$ & $0.112(6)$ & $0.026(4)$ & $0.087(5)$ & $0.009(4)$ & $0.009(5)$ & $-0.004(4)$ \\
\hline$C(11 \mathrm{~A})$ & $2 a$ & $0.6467(7)$ & $0.185(1)$ & $0.8064(4)$ & $0.085(7)$ & $0.034(6)$ & $0.066(6)$ & $-0.004(5)$ & $0.021(5)$ & $-0.001(5)$ \\
\hline$O(4 A)$ & $2 a$ & $0.5927(5)$ & $0.163(1)$ & $0.8427(3)$ & $0.104(5)$ & $0.043(4)$ & $0.059(4)$ & $0.004(4)$ & $0.017(4)$ & $0.004(4)$ \\
\hline$C(12 A)$ & $2 a$ & $0.6247(9)$ & $0.149(2)$ & $0.9002(5)$ & $0.14(1)$ & $0.047(7)$ & $0.059(6)$ & $0.000(8)$ & $0.003(7)$ & $0.009(6)$ \\
\hline$C(22 \mathrm{~A})$ & $2 a$ & $0.562(1)$ & $0.106(2)$ & $0.9315(6)$ & $0.16(2)$ & $0.043(8)$ & $0.09(1)$ & $-0.012(9)$ & $0.03(1)$ & $0.010(7)$ \\
\hline $\mathrm{O}(22 \mathrm{~A})$ & $2 a$ & $0.5274(7)$ & $-0.053(1)$ & $0.9156(5)$ & $0.16(1)$ & $0.060(7)$ & $0.148(9)$ & $-0.011(6)$ & $0.059(8)$ & $0.015(7)$ \\
\hline $\mathrm{C}(32 \mathrm{~A})$ & $2 a$ & $0.501(1)$ & $0.252(2)$ & $0.9268(6)$ & $0.15(1)$ & $0.039(7)$ & $0.092(9)$ & $0.010(8)$ & $0.048(9)$ & $0.017(7)$ \\
\hline$O(32 \mathrm{~A})$ & $2 a$ & $0.4456(6)$ & $0.221(1)$ & $0.9612(4)$ & $0.138(8)$ & $0.081(7)$ & $0.128(7)$ & $0.000(7)$ & $0.076(6)$ & $0.023(7)$ \\
\hline $\mathrm{C}(42 \mathrm{~A})$ & $2 a$ & $0.546(1)$ & $0.415(2)$ & $0.9453(6)$ & $0.14(1)$ & $0.055(9)$ & $0.09(1)$ & $0.030(9)$ & $0.039(9)$ & $0.023(8)$ \\
\hline $\mathrm{O}(42 \mathrm{~A})$ & $2 a$ & $0.4920(6)$ & $0.554(1)$ & $0.9358(5)$ & $0.127(9)$ & $0.055(6)$ & $0.18(1)$ & $0.017(6)$ & $0.052(8)$ & $0.003(7)$ \\
\hline $\mathrm{C}(52 \mathrm{~A})$ & $2 a$ & $0.6122(9)$ & $0.446(2)$ & $0.9146(5)$ & $0.12(1)$ & $0.040(7)$ & $0.072(8)$ & $0.011(7)$ & $0.019(7)$ & $0.011(6)$ \\
\hline$O(52 A)$ & $2 a$ & $0.6630(5)$ & $0.301(1)$ & $0.9204(3)$ & $0.104(6)$ & $0.047(5)$ & $0.069(5)$ & $0.004(4)$ & $0.004(4)$ & $-0.008(4)$ \\
\hline$C(62 A)$ & $2 a$ & $0.6597(8)$ & $0.590(2)$ & $0.9425(5)$ & $0.12(1)$ & $0.049(8)$ & $0.080(8)$ & $-0.003(7)$ & $0.022(7)$ & $-0.006(6)$ \\
\hline$O(62 A)$ & $2 a$ & $0.7212(5)$ & $0.632(1)$ & $0.9101(3)$ & $0.083(5)$ & $0.061(5)$ & $0.088(5)$ & $-0.011(5)$ & $0.019(4)$ & $0.002(5)$ \\
\hline$S(2 A)$ & $2 a$ & $0.8021(3)$ & $0.7034(5)$ & $0.9411(2)$ & $0.127(3)$ & $0.048(2)$ & $0.106(3)$ & $-0.007(2)$ & $0.011(2)$ & $0.004(2)$ \\
\hline$O(9 A)$ & $2 a$ & $0.7884(7)$ & $0.840(1)$ & $0.9787(4)$ & $0.17(1)$ & $0.049(6)$ & $0.121(8)$ & $-0.009(6)$ & $0.019(7)$ & $-0.024(6)$ \\
\hline$O(10 A)$ & $2 a$ & $0.8461(7)$ & $0.739(1)$ & $0.8963(4)$ & $0.18(1)$ & $0.081(8)$ & $0.109(7)$ & $-0.021(8)$ & $0.052(7)$ & $0.032(6)$ \\
\hline$C(7 A)$ & $2 a$ & $0.8446(8)$ & $0.531(2)$ & $0.9792(6)$ & $0.091(9)$ & $0.047(7)$ & $0.10(1)$ & $-0.009(7)$ & $0.021(7)$ & $-0.007(7)$ \\
\hline
\end{tabular}


Table 3. Continued.

\begin{tabular}{|c|c|c|c|c|c|c|c|c|c|c|}
\hline Atom & Site & $\boldsymbol{x}$ & $y$ & $z$ & $U_{11}$ & $U_{22}$ & $U_{33}$ & $U_{12}$ & $U_{13}$ & $U_{23}$ \\
\hline$C(8 A)$ & $2 a$ & $0.851(1)$ & $0.379(2)$ & $0.9530(7)$ & $0.13(1)$ & $0.06(1)$ & $0.12(1)$ & $-0.002(9)$ & $0.03(1)$ & $-0.01(1)$ \\
\hline$C(9 A)$ & $2 a$ & $0.885(1)$ & $0.240(3)$ & $0.9851(9)$ & $0.11(1)$ & $0.10(1)$ & $0.16(2)$ & $0.02(1)$ & $0.04(1)$ & $0.03(1)$ \\
\hline$C(10 A)$ & $2 a$ & $0.907(1)$ & $0.252(3)$ & $1.041(1)$ & $0.14(2)$ & $0.09(1)$ & $0.14(2)$ & $0.03(1)$ & $-0.01(1)$ & $0.02(1)$ \\
\hline $\mathrm{C}(91 \mathrm{~A})$ & $2 a$ & $0.896(1)$ & $0.396(3)$ & $1.0664(7)$ & $0.14(1)$ & $0.11(2)$ & $0.09(1)$ & $-0.00(1)$ & $-0.00(1)$ & $0.04(1)$ \\
\hline$C(13 A)$ & $2 a$ & $0.864(1)$ & $0.543(2)$ & $1.0379(7)$ & $0.14(1)$ & $0.08(1)$ & $0.10(1)$ & $0.00(1)$ & $0.02(1)$ & $0.01(1)$ \\
\hline $\mathrm{C}(2 \mathrm{MA})$ & $2 a$ & $0.940(1)$ & $0.088(3)$ & $1.074(1)$ & $0.14(2)$ & $0.14(2)$ & $0.24(2)$ & $0.03(2)$ & $0.03(2)$ & $0.11(2)$ \\
\hline $\mathrm{C}(1 \mathrm{MB})$ & $2 a$ & $0.078(1)$ & $1.240(3)$ & $0.5841(8)$ & $0.11(1)$ & $0.12(2)$ & $0.19(2)$ & $-0.02(1)$ & $0.03(1)$ & $-0.09(2)$ \\
\hline $\mathrm{C}(1 \mathrm{~B})$ & $2 a$ & $0.0968(8)$ & $1.091(2)$ & $0.5467(8)$ & $0.076(8)$ & $0.06(1)$ & $0.15(2)$ & $0.002(7)$ & $0.025(9)$ & $-0.03(1)$ \\
\hline$C(2 B)$ & $2 a$ & $0.098(1)$ & $0.923(2)$ & $0.5667(7)$ & $0.17(2)$ & $0.08(1)$ & $0.10(1)$ & $0.01(1)$ & $0.05(1)$ & $-0.02(1)$ \\
\hline$C(3 B)$ & $2 a$ & $0.116(1)$ & $0.792(2)$ & $0.5334(7)$ & $0.18(2)$ & $0.07(1)$ & $0.12(1)$ & $0.04(1)$ & $0.06(1)$ & $0.02(1)$ \\
\hline$C(4 B)$ & $2 a$ & $0.1302(7)$ & $0.824(2)$ & $0.4807(6)$ & $0.083(8)$ & $0.047(7)$ & $0.099(9)$ & $0.023(6)$ & $0.031(7)$ & $0.011(7)$ \\
\hline $\mathrm{C}(5 \mathrm{~B})$ & $2 a$ & $0.1289(9)$ & $0.992(2)$ & $0.4618(6)$ & $0.13(1)$ & $0.043(8)$ & $0.10(1)$ & $0.008(8)$ & $0.047(9)$ & $0.015(7)$ \\
\hline $\mathrm{C}(6 \mathrm{~B})$ & $2 a$ & $0.1104(7)$ & $1.121(2)$ & $0.4947(7)$ & $0.086(9)$ & $0.055(9)$ & $0.12(1)$ & $-0.009(7)$ & $0.018(8)$ & $0.009(9)$ \\
\hline$S(1 B)$ & $2 a$ & $0.1464(2)$ & $0.6587(4)$ & $0.4374(2)$ & $0.094(2)$ & $0.057(2)$ & $0.102(2)$ & $0.018(2)$ & $0.018(2)$ & $0.004(2)$ \\
\hline $\mathrm{O}(7 \mathrm{~B})$ & $2 a$ & $0.1085(7)$ & $0.507(1)$ & $0.4518(6)$ & $0.116(8)$ & $0.046(6)$ & $0.23(1)$ & $-0.023(6)$ & $0.050(9)$ & $-0.004(8)$ \\
\hline$O(8 B)$ & $2 a$ & $0.1347(6)$ & $0.713(2)$ & $0.3812(4)$ & $0.131(8)$ & $0.117(9)$ & $0.084(6)$ & $0.045(7)$ & $0.014(5)$ & $0.019(6)$ \\
\hline $\mathrm{O}(63 \mathrm{~B})$ & $2 a$ & $0.2364(4)$ & $0.638(1)$ & $0.4577(3)$ & $0.083(5)$ & $0.039(4)$ & $0.103(5)$ & $0.026(4)$ & $0.022(4)$ & $-0.005(4)$ \\
\hline$C(63 B)$ & $2 a$ & $0.2774(7)$ & $0.502(2)$ & $0.4336(5)$ & $0.088(8)$ & $0.043(7)$ & $0.074(7)$ & $0.018(6)$ & $0.017(6)$ & $0.000(6)$ \\
\hline $\mathrm{C}(53 \mathrm{~B})$ & $2 a$ & $0.3267(6)$ & $0.403(1)$ & $0.4811(4)$ & $0.084(7)$ & $0.025(5)$ & $0.060(6)$ & $0.001(5)$ & $0.017(5)$ & $0.008(5)$ \\
\hline $\mathrm{O}(53 \mathrm{~B})$ & $2 a$ & $0.2711(4)$ & $0.317(1)$ & $0.5097(3)$ & $0.079(4)$ & $0.046(4)$ & $0.064(4)$ & $-0.007(4)$ & $0.012(3)$ & $0.008(4)$ \\
\hline$C(43 B)$ & $2 a$ & $0.3800(7)$ & $0.272(1)$ & $0.4610(4)$ & $0.090(7)$ & $0.018(5)$ & $0.066(6)$ & $0.010(5)$ & $0.015(5)$ & $0.000(5)$ \\
\hline$O(43 B)$ & $2 a$ & $0.4317(5)$ & $0.352(1)$ & $0.4300(3)$ & $0.091(5)$ & $0.052(5)$ & $0.061(4)$ & $0.005(4)$ & $0.018(4)$ & $0.011(4)$ \\
\hline$C(33 B)$ & $2 a$ & $0.4235(7)$ & $0.179(1)$ & $0.5105(4)$ & $0.088(7)$ & $0.025(5)$ & $0.071(6)$ & $0.008(5)$ & $0.013(5)$ & $-0.001(5)$ \\
\hline $\mathrm{O}(33 \mathrm{~B})$ & $2 a$ & $0.4703(5)$ & $0.044(1)$ & $0.4959(3)$ & $0.122(7)$ & $0.048(5)$ & $0.068(4)$ & $0.037(5)$ & $0.022(5)$ & $0.000(4)$ \\
\hline$C(23 B)$ & $2 a$ & $0.3649(7)$ & $0.100(1)$ & $0.5450(4)$ & $0.096(8)$ & $0.019(5)$ & $0.063(6)$ & $-0.007(5)$ & $-0.001(5)$ & $0.002(4)$ \\
\hline $\mathrm{O}(23 \mathrm{~B})$ & $2 a$ & $0.4049(5)$ & $0.0251(9)$ & $0.5951(3)$ & $0.123(7)$ & $0.026(4)$ & $0.063(4)$ & $-0.004(4)$ & $0.014(4)$ & $0.002(3)$ \\
\hline$C(13 B)$ & $2 a$ & $0.3074(6)$ & $0.232(1)$ & $0.5579(4)$ & $0.084(7)$ & $0.015(5)$ & $0.063(6)$ & $-0.002(5)$ & $0.005(5)$ & $-0.001(4)$ \\
\hline$O(4 B)$ & $2 a$ & $0.3493(4)$ & $0.3494(8)$ & $0.5978(3)$ & $0.075(4)$ & $0.027(4)$ & $0.074(4)$ & $-0.002(3)$ & $0.023(4)$ & $-0.003(3)$ \\
\hline$C(14 B)$ & $2 a$ & $0.2945(7)$ & $0.461(1)$ & $0.6188(5)$ & $0.108(9)$ & $0.030(5)$ & $0.076(7)$ & $0.004(6)$ & $0.056(7)$ & $0.008(5)$ \\
\hline $\mathrm{C}(24 \mathrm{~B})$ & $2 a$ & $0.3402(7)$ & $0.613(1)$ & $0.6452(5)$ & $0.103(8)$ & $0.019(5)$ & $0.075(7)$ & $-0.002(5)$ & $0.022(6)$ & $-0.001(5)$ \\
\hline $\mathrm{O}(24 \mathrm{~B})$ & $2 a$ & $0.3861(5)$ & $0.6857(9)$ & $0.6067(3)$ & $0.124(6)$ & $0.029(4)$ & $0.066(4)$ & $-0.011(4)$ & $0.028(4)$ & $0.010(3)$ \\
\hline$C(34 B)$ & $2 a$ & $0.3919(7)$ & $0.570(1)$ & $0.7012(5)$ & $0.095(8)$ & $0.026(5)$ & $0.070(7)$ & $0.002(5)$ & $0.018(6)$ & $-0.002(5)$ \\
\hline$O(34 B)$ & $2 a$ & $0.4221(5)$ & $0.728(1)$ & $0.7273(3)$ & $0.137(7)$ & $0.058(6)$ & $0.077(5)$ & $-0.030(6)$ & $0.031(5)$ & $-0.014(5)$ \\
\hline$C(44 B)$ & $2 a$ & $0.3508(8)$ & $0.472(1)$ & $0.7388(5)$ & $0.109(9)$ & $0.032(6)$ & $0.066(7)$ & $-0.006(6)$ & $0.010(6)$ & $0.005(5)$ \\
\hline$O(44 B)$ & $2 a$ & $0.4026(6)$ & $0.414(1)$ & $0.7863(3)$ & $0.115(7)$ & $0.085(7)$ & $0.078(6)$ & $-0.016(6)$ & $0.018(5)$ & $0.004(5)$ \\
\hline $\mathrm{C}(54 \mathrm{~B})$ & $2 a$ & $0.3127(7)$ & $0.319(1)$ & $0.7069(5)$ & $0.084(7)$ & $0.040(6)$ & $0.079(7)$ & $0.002(5)$ & $0.035(6)$ & $0.006(6)$ \\
\hline $\mathrm{O}(54 \mathrm{~B})$ & $2 a$ & $0.2572(5)$ & $0.375(1)$ & $0.6593(3)$ & $0.087(5)$ & $0.047(5)$ & $0.072(4)$ & $-0.004(4)$ & $0.027(4)$ & $-0.005(4)$ \\
\hline $\mathrm{C}(64 \mathrm{~B})$ & $2 a$ & $0.2670(8)$ & $0.198(2)$ & $0.7381(5)$ & $0.104(9)$ & $0.048(7)$ & $0.098(8)$ & $0.006(7)$ & $0.051(7)$ & $0.002(7)$ \\
\hline$O(64 B)$ & $2 a$ & $0.2030(6)$ & $0.297(1)$ & $0.7562(4)$ & $0.135(8)$ & $0.043(5)$ & $0.109(6)$ & $0.002(5)$ & $0.059(6)$ & $0.021(5)$ \\
\hline$S(2 B)$ & $2 a$ & $0.1580(3)$ & $0.2074(5)$ & $0.7996(2)$ & $0.149(4)$ & $0.060(2)$ & $0.115(3)$ & $-0.023(3)$ & $0.064(3)$ & $-0.013(2)$ \\
\hline$O(9 B)$ & $2 a$ & $0.0999(8)$ & $0.327(2)$ & $0.8080(5)$ & $0.19(1)$ & $0.085(8)$ & $0.19(1)$ & $-0.040(8)$ & $0.13(1)$ & $-0.052(9)$ \\
\hline$O(10 B)$ & $2 a$ & $0.2154(7)$ & $0.152(2)$ & $0.8457(4)$ & $0.104(8)$ & $0.064(8)$ & $0.054(6)$ & $-0.012(7)$ & $0.014(6)$ & $0.007(6)$ \\
\hline$C(7 B)$ & $2 a$ & $0.1117(8)$ & $0.029(2)$ & $0.7648(6)$ & $0.088(9)$ & $0.043(7)$ & $0.11(1)$ & $-0.003(7)$ & $0.032(8)$ & $0.009(7)$ \\
\hline$C(8 B)$ & $2 a$ & $0.044(1)$ & $0.038(2)$ & $0.7280(7)$ & $0.10(1)$ & $0.07(1)$ & $0.13(1)$ & $-0.015(9)$ & $0.03(1)$ & $0.00(1)$ \\
\hline $\mathrm{C}(9 \mathrm{~B})$ & $2 a$ & $0.012(1)$ & $-0.106(2)$ & $0.7006(6)$ & $0.12(1)$ & $0.09(1)$ & $0.10(1)$ & $-0.02(1)$ & $0.016(9)$ & $-0.02(1)$ \\
\hline $\mathrm{C}(10 \mathrm{~B})$ & $2 a$ & $0.050(1)$ & $-0.262(2)$ & $0.7085(8)$ & $0.12(1)$ & $0.06(1)$ & $0.15(2)$ & $-0.004(9)$ & $0.05(1)$ & $0.00(1)$ \\
\hline$C(11 B)$ & $2 a$ & $0.1194(9)$ & $-0.270(2)$ & $0.7429(8)$ & $0.084(9)$ & $0.06(1)$ & $0.18(2)$ & $-0.007(8)$ & $0.00(1)$ & $0.01(1)$ \\
\hline$C(12 B)$ & $2 a$ & $0.1515(9)$ & $-0.126(2)$ & $0.7733(7)$ & $0.090(9)$ & $0.07(1)$ & $0.14(1)$ & $-0.006(8)$ & $-0.005(9)$ & $0.01(1)$ \\
\hline $\mathrm{C}(2 \mathrm{MB})$ & $2 a$ & $0.012(1)$ & $-0.413(2)$ & $0.6757(8)$ & $0.15(2)$ & $0.07(1)$ & $0.20(2)$ & $-0.03(1)$ & $0.03(1)$ & $-0.06(1)$ \\
\hline Ow(1) & $2 a$ & $0.5296(5)$ & $0.501(1)$ & $0.6152(3)$ & $0.119(6)$ & $0.041(5)$ & $0.068(5)$ & $-0.015(4)$ & $0.018(4)$ & $0.000(4)$ \\
\hline Ow(2) & $2 a$ & $0.339(2)$ & $0.000(5)$ & $0.911(1)$ & $0.25(2)$ & $0.42(5)$ & $0.30(3)$ & $0.06(3)$ & $0.03(2)$ & $0.06(3)$ \\
\hline $\operatorname{Om}(1)$ & $2 a$ & $0.396(1)$ & $-0.090(3)$ & $0.8190(8)$ & $0.23(2)$ & $0.19(2)$ & $0.20(2)$ & $0.03(2)$ & $0.01(1)$ & $-0.05(2)$ \\
\hline $\mathrm{Cm}(1)$ & $2 a$ & $0.453(2)$ & $-0.223(4)$ & $0.846(2)$ & $0.56(9)$ & $0.11(3)$ & $0.59(9)$ & $-0.02(4)$ & $0.21(8)$ & $0.05(4)$ \\
\hline $\operatorname{Om}(2)$ & $2 a$ & $0.666(2)$ & $0.053(6)$ & $1.1205(8)$ & $0.40(4)$ & $0.57(7)$ & $0.14(1)$ & $-0.12(5)$ & $-0.04(2)$ & $0.06(3)$ \\
\hline $\mathrm{Cm}(2)$ & $2 a$ & $0.713(2)$ & $0.040(8)$ & $1.072(2)$ & $0.21(3)$ & $0.46(8)$ & $0.29(4)$ & $0.08(4)$ & $0.08(3)$ & $0.07(5)$ \\
\hline
\end{tabular}

Acknowledgments. We gratefully acknowledge M.U.R.S.T., the Ministry of University, and Scientific and Technological Research, and The National Research Council of Italy, C.N.R. for their continous and generous support. This work was partially supported by CNR grant n 104110.12 .9705108 .

\section{References}

1. Cucinotta, V.; Grasso, G.; Vecchio, G.: From capped to three-dimensional cyclodextrins: the first example of a new class of receptors by trehalose capping of $\beta$-cyclodextrin. J. Incl. Phenom. 31 (1998) 43-55.

2. Brochere-Ferreol, G.; Polonsky, J.: Sur la synthese de substances a actyvite de cord factor. Syntheses de diesters de trehalose en position 6,6'. Bull. Soc. Chim. France (1958) 714-717.
3. Frenz, B. A. \& Associated Int.: Structure Determination Package. A System of Computer Programs. College Station, TX, and Enraf Nonius, Delft, Holland 1986.

4. Altomare, A.; Cascarano, G.; Giacovazzo, C.; Guagliardi, A.; Burla, M. C.; Polidori, G. C.; Camalli, M.: SIR92, a program for automatic solution of crystal structures by direct methods. J. Appl. Crystallogr. 27 (1994) 435.

5. Sheldrick, G. M.: SHELXL-93, a program for refining crystal structures. University of Göttingen, Germany 1993.

6. Spek, A. L.: The Euclid package. In: Computional crystallography (Ed. D. Syre), p. 528. Oxford Clerendon Press 1982. 



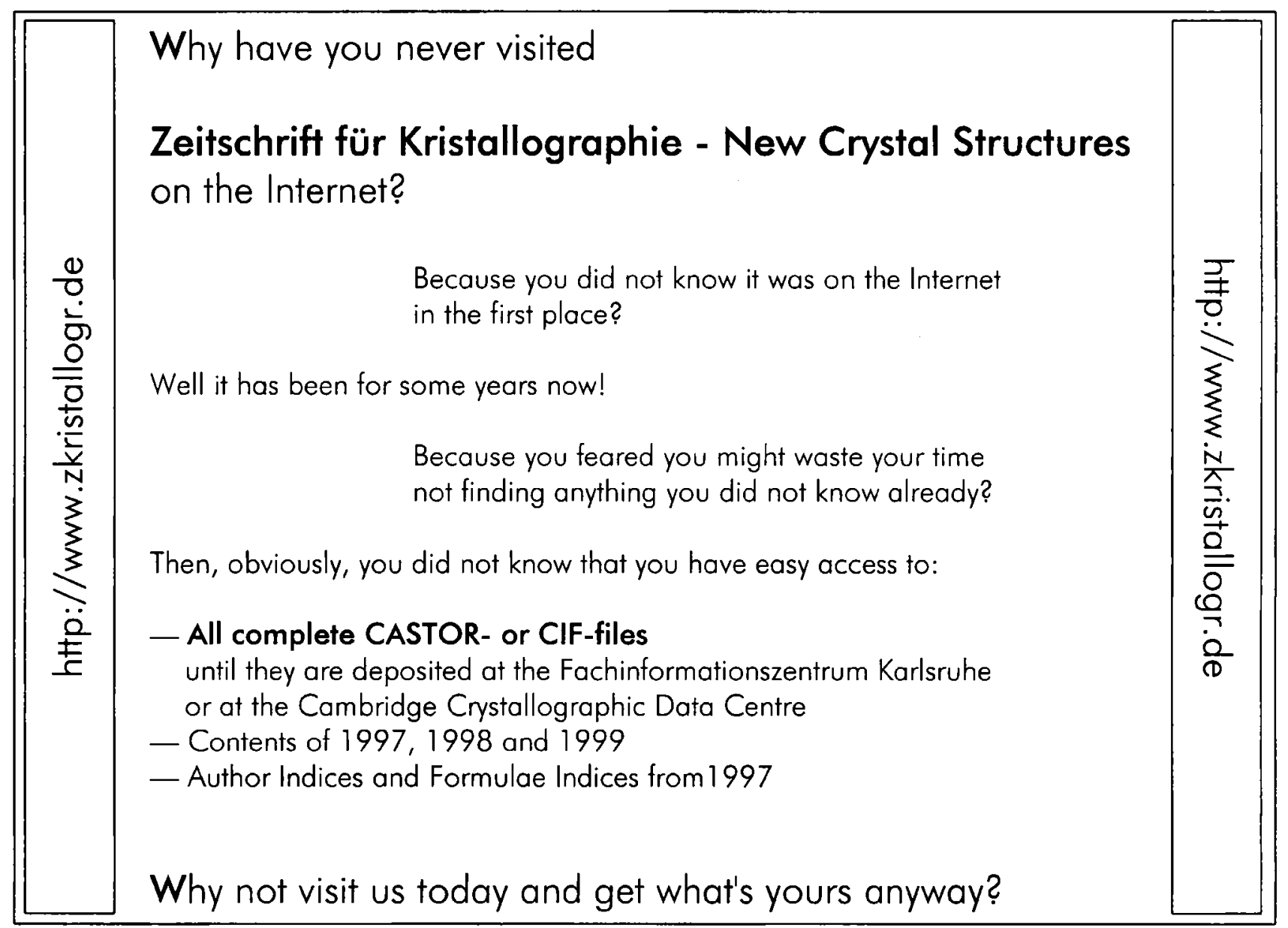

